\title{
Probing sterile neutrinos in $B(D)$ meson decays at Belle II (BESIII)
}

\author{
C. S. Kim ${ }^{1,2, a}$, Youngjoon Kwon ${ }^{1, b}$, Donghun Lee ${ }^{1, \mathrm{c}}$, Sechul Oh $^{3, \mathrm{~d}}$, Dibyakrupa Sahoo ${ }^{1, \mathrm{e}} \mathbb{C}_{\mathbb{C}}$ \\ ${ }^{1}$ Department of Physics and IPAP, Yonsei University, Seoul 03722, South Korea \\ 2 Institute of High Energy Physics, Dongshin University, Naju 58245, South Korea \\ ${ }^{3}$ University College, Yonsei University, Incheon 21983, South Korea
}

Received: 24 May 2020 / Accepted: 30 July 2020 / Published online: 12 August 2020

(C) The Author(s) 2020

\begin{abstract}
We present, how a systematic study of $B \rightarrow$ $D \ell N(D \rightarrow K \ell N)$ decays with $\ell=\mu, \tau$, at Belle II (BESIII) can provide unambiguous signature of a heavy neutrino $N$ and/or constrain its mixing with active neutrinos $v_{\ell}$, which is parameterized by $\left|U_{\ell N}\right|^{2}$. Our constraint on $\left|U_{\mu N}\right|^{2}$ that can be achieved from the full Belle II data is comparable with what can be obtained from the much larger data set of the upgraded LHCb. Additionally, our method offers better constraint on $\left|U_{\mu N}\right|^{2}$ for mass of sterile neutrino $m_{N}<2 \mathrm{GeV}$. We can also probe the Dirac and Majorana nature of $N$ by observing the sequential decay of $N$, including suppression from observation of a displaced vertex as well as helicity flip, for Majorana $N$.
\end{abstract}

\section{Introduction}

In many new physics theories implementing the see-saw mechanisms, there are one or more heavier cousins of the active flavor neutrinos $v_{\ell}(\ell=e, \mu, \tau)$ which do not have any interaction with Standard Model (SM) particles except mixing with the active neutrinos. These heavy neutrinos are named as sterile neutrinos which can be either Dirac or Majorana fermions. Among the varieties of new physics scenarios where sterile neutrinos appear, the original seesaw mechanism [1-6] predicts their mass to be much larger than $1 \mathrm{TeV}$. In other seesaw models heavy neutrinos can have mass in a very large range, from $\sim 0.1$ to $1 \mathrm{TeV}$ [7-16], or close to about $1 \mathrm{GeV}$ [17-32], or even at $\mathrm{keV}$ scale [33] or eV scale [34]. The mixing parameters, $U_{\ell N}$, which describe the

\footnotetext{
a e-mail: cskim@yonsei.ac.kr

be-mail: yjkwon63@yonsei.ac.kr

c e-mail: donghun.lee@yonsei.ac.kr

de-mail: scohph@yonsei.ac.kr

e e-mail: sahoodibya@yonsei.ac.kr (corresponding author)
}

strength of mixing between a sterile (heavy) neutrino $N$ with the SM flavor (light) neutrinos $\nu_{\ell}$ are constrained by various experimental data depending on the mass of $N$ (see Refs. [35,36] for further details and references).

The neutrinos are the only fermions which can be their own anti-particles, i.e. behave as Majorana fermions. Ascertaining their Dirac or Majorana nature is one of the most important issues in neutrino physics. It is well known that Dirac neutrinos can participate only in the lepton number conserving (LNC) processes, while Majorana neutrinos can get involved in both lepton number violating (LNV) and LNC processes. Therefore, to investigate the Majorana nature of neutrinos, many attempts have been made at studying various LNV processes, including neutrinoless double beta decay $(0 \nu \beta \beta)$ ([37], for early reviews see [38-40], for recent reviews see $[41,42])$, specific LNV processes at LHC [1517,43-63], LNV $\tau$ lepton decays [64-69], and LNV rare meson decays [70-87], e.g. rare LNV decays of $K, D_{(s)}$, $B_{(c)}$ mesons have been studied extensively in literature. In particular, semileptonic decays such as $B \rightarrow D \ell \ell \pi$ and $B \rightarrow \ell \ell \pi$ were explored in Refs. $[86,87]$ to not only distinguish between Dirac and Majorana signatures, but also constrain $\left|U_{\ell N}\right|^{2}$, without considering, in detail, the feasibility of observation of these decays inside a sizable detector including the helicity flip for Majorana case.

Our main significant result in this paper is the stringent constraint that can be put on $\left|U_{\ell N}\right|^{2}$, especially on $\left|U_{\mu N}\right|^{2}$, from non-observation of the decays $B \rightarrow D \ell N$, without considering the sequential decay of $N$. This simple strategy has, however, remained unexplored in the currently existing literature. Instead of considering two-body leptonic decays $B^{+} \rightarrow \ell^{+} N$, similar to existing studies on $\pi^{+}\left(\right.$or $\left.K^{+}\right) \rightarrow \ell^{+} N$ which look for mono-energetic $\ell^{+}$to constrain $\left|U_{\ell N}\right|^{2}$ [88-107], we have considered the threebody semileptonic decays $B \rightarrow D \ell N$ which have bigger branching ratios in a larger mass range. The reach of our 
study to constrain $\left|U_{\mu N}\right|^{2}$ and $\left|U_{\tau N}\right|^{2}$ is better by an order of magnitude from existing experimental constraints in certain mass ranges of interest. Interestingly, our constraint on $\left|U_{\mu N}\right|^{2}$ obtained by considering only $\sim 4.8 \times 10^{8}$ events of fully reconstructed $B \rightarrow D \mu N$ decays at Belle II [108] is comparable with the constraint achievable from $4.8 \times 10^{12}$ events of $B \rightarrow D \mu \mu \pi$ decays at upgraded LHCb [36]. Although the missing sterile neutrino search gives the stringent constraint on $\left|U_{\ell N}\right|^{2}$, it can not distinguish Dirac and Majorana neutrinos. Therefore, we also study the sequential decay of $N$ with a displaced vertex signature for probing its Majorana nature, and consider the important but otherwise overlooked effect of helicity flip for sterile neutrinos. Despite the suppression coming from observation of displaced vertices as well as the helicity flip, we find that heavier and less energetic neutrinos have a bigger chance of decaying inside a detector with decay length $\leqslant 1 \mathrm{~m}$, provided they exist. Finally, we present an estimate of $\left|U_{\mu N}\right|^{2}$ in the case of observation of LNC $B \rightarrow D \mu^{+} \mu^{-} \pi^{+}$decay in Belle II. The LNV mode $B \rightarrow D \mu^{+} \mu^{+} \pi^{-}$receives additional suppression from helicity flip.

This paper is organized as follows. In Sect. 2 we provide the logical basis for considering the decays $B \rightarrow D \ell N$ or $D \rightarrow K \ell N$ and the advantages they offer over choosing any other processes. In Sect. 3 we show how well the mixing parameters $\left|U_{\mu N}\right|^{2}$ and $\left|U_{\tau N}\right|^{2}$ can be probed by using these decays at Belle II. We also provide a short discussion, via example, on possible SM background processes and how they can be distinguished from the signal events. This is followed by a discussion in Sect. 4 on how the Majorana nature of neutrino could be probed, and whether it is possible to do such a study. Finally we conclude in Sect. 5 highlighting the important features of our paper.

\section{Choosing appropriate production modes}

We aim to find a process that would (1) unambiguously probe the presence of sterile neutrino $N$, free from any other new physics possibilities, and (2) constrain the mixing of $N$ with active neutrinos. In this regard, we find it helpful to keep in mind the following four cardinal aspects of sterile neutrino. Any candidate for sterile neutrino would have (i) electric charge $=0$, (ii) $\operatorname{spin}=1 / 2$, (iv) mass $>0$, and (v) possibly long life-time. The fact that the sterile neutrino would most likely remain undetected at its point of production, is in fact the experimental consequence of its possibly long life-time and electrically neutral nature. This manifests as 'missing momentum' in any process which would have sterile neutrino(s) in the final state, just like the case with ordinary active neutrino(s) in any final state.

As examples of processes with 'missing momentum' we can consider meson decays such as $B \rightarrow D^{(*)} \mu X, D \rightarrow$
$K^{(*)} \mu X, B \rightarrow K X$ etc., where $X$ denotes missing (i.e. undetected) particle(s) other than active neutrinos. A simple analysis of spin would suffice to illustrate the fact that for decays such as $B \rightarrow D^{*} \mu X$ and $D \rightarrow K^{*} \mu X$, the spin of $X$ is ambiguous: it could be $1 / 2$ or $3 / 2$. Similarly, in the decay $B \rightarrow K X$, the 'missing' $X$ would indeed be made of (at least) two invisible particles and their individual spins could be $0,1 / 2$ or $3 / 2$. In order to avoid such ambiguities, we shall refrain from considering these types of decays in this paper. Thus we are left to consider decays of the type $B \rightarrow D \mu N$ and $D \rightarrow K \mu N$ as decay modes suitable for discovery of sterile neutrino $N$.

It should be noted that the literature dealing with sterile heavy neutrino searches is replete with LNC and LNV processes mediated by sterile neutrino $N$, such as $B \rightarrow D \ell \ell \pi$, $B \rightarrow \ell \ell \pi, \tau \rightarrow \pi \ell_{1} \ell_{2} v$ etc. where $\ell, \ell_{1,2}=e, \mu$. These processes, can also take place via other new physics possibilities, such as exotic scalars, vectors or lepto-quarks. Nevertheless, the LNV processes mediated by $N$ constitute the only known reliable methodology to probe the conjectured Majorana nature of $N$. Notwithstanding the importance, these neutrino mediated decays are suppressed from associated displaced vertices, branching ratio of sequential decay of $N$ as well as helicity flip of $N$ (relevant in case of LNV modes only). Therefore, when concerned with the discovery prospect of sterile neutrino, we shall refrain from considering the sequential decay of $N$. Once an unambiguous signature of existence of sterile neutrino $N$ is obtained, study of its Dirac or Majorana nature becomes highly relevant, and in this context we would consider the feasibility of the complete LNC and LNV modes which take into account the sequential decay of $N$.

Let us now analyze the decays $B \rightarrow D \mu N$ (or $D \rightarrow$ $K \mu N$ ) with $N$ remaining undetected in the detector. We are interested in the scenario where there is one light sterile neutrino with mass $m_{N} \leqslant 3.3 \mathrm{GeV}$. The criteria of massive sterile neutrino helps us to eliminate background events to our processes. The decay $B \rightarrow D \mu N$, for example, can receive background from the decay $B \rightarrow D \mu v_{\mu} \nu \bar{v}$ or $B \rightarrow D^{*}\left(\rightarrow D \pi_{\text {soft }} / \gamma_{\text {soft }}\right) \mu \nu$, where the soft pion $\left(\pi_{\text {soft }}\right)$ or soft photon $\left(\gamma_{\text {soft }}\right)$ arising from sequential decay of $D^{*}$ are not detected by the detector. However, the invariant mass obtained from the missing 4-momenta of background process would vary significantly from one event to another unlike the signal case which would be centered about the fixed mass of $N$.

In order to obtain the 'missing mass' $\left(m_{\text {miss }}\right)$ in the processes, say $B \rightarrow D \mu+$ 'missing' that includes the signal events for $B \rightarrow D \mu N$ also, we need to know the 4-momenta of initial $B$ meson $\left(p_{B}\right)$, final $D$ meson $\left(p_{D}\right)$ and $\mu\left(p_{\mu}\right)$ :

$m_{\text {miss }} \equiv \sqrt{p_{\text {miss }}^{2}}=\sqrt{\left(p_{B}-p_{D}-p_{\mu}\right)^{2}}$. 
For signal events only $m_{\text {miss }}=m_{N}$. This methodology is applicable in experiments such as Belle II or BESIII where $B$ and $D$ mesons are pair produced along with $\bar{B}$ and $\bar{D}$ from the decays $\Upsilon(4 S) \rightarrow B \bar{B}$ and $\psi(3770) \rightarrow D \bar{D}$ respectively, and the 4-momentum of $\bar{B}, \bar{D}$ can be precisely measured by full hadronic reconstruction. It should be, therefore, clear that our methodology is not applicable for experiments such as LHCb where the initial 4-momentum of the $B$ or $D$ meson can not be inferred without measuring the 4-momenta of the final particles arising from the $B$ or $D$ meson decay. Furthermore, the minimum value of mass $m_{N} \neq 0$ that can be probed in our approach is, therefore, constrained only by the experimental accuracy of measurement of 4-momenta of $B$, $D$ and $\mu$. In the next section we would provide a numerical comparison of a few observables (including $m_{N}$ ), for the SM background decay $B \rightarrow D \mu \nu \pi_{\text {soft }} / \gamma_{\text {soft }}$ and the signal decay $B \rightarrow D \mu N$, specifically in context of Belle II. It is important to note that $N$ may or may not decay inside a detector, depending on its mass, energy and the size of the detector. If it decays inside the detector, with noticeable displaced vertex, we can not only measure its 4-momentum directly from its decay products, but also probe its Dirac or Majorana nature as well as veto any background events for the decays under consideration.

Note that the decays $B^{+} \rightarrow \tau^{+} N$ and $B \rightarrow D \tau N$, where the 4-momentum of the tau lepton is reconstructed from its further sequential decay, are less promising for our study, due to the presence of at least one neutrino (or antineutrino) in the final state of all tau decays. Nevertheless, taking into account that the tau 4-momentum could be measured accurately with a smaller probability, we shall constrain $\left|U_{\tau N}\right|^{2}$ from $B \rightarrow$ $D \tau N$.

\section{Determining or constraining the value of $\left|U_{\mu N}\right|^{2}$ and $\left|U_{\tau N}\right|^{2}$}

The branching ratios of all the decay modes under our consideration are directly proportional to the appropriate activesterile mixing parameter $\left|U_{\ell N}\right|^{2}$. We obtain the canonical branching ratio of a decay, e.g. $B \rightarrow D \ell N$, by factoring out $\left|U_{\ell N}\right|^{2}$ from the theoretically calculable branching ratio [86],

$\underline{\operatorname{Br}}(B \rightarrow D \ell N)=\frac{\operatorname{Br}(B \rightarrow D \ell N)}{\left|U_{\ell N}\right|^{2}}$.

Given the value of canonical branching ratio $\underline{\operatorname{Br}}(B \rightarrow D \ell N)$, the number of such decays observable in the detector $\left(N_{B \rightarrow D \ell N}\right)$ and the total number of fully reconstructed parent particles $\left(N_{B}\right)$, we can estimate $\left|U_{\ell N}\right|^{2}$ by

$$
\left|U_{\ell N}\right|^{2}=\frac{N_{B \rightarrow D \ell N}}{N_{B} \times \epsilon_{D} \times \epsilon_{\ell} \times \underline{\operatorname{Br}}(B \rightarrow D \ell N)},
$$

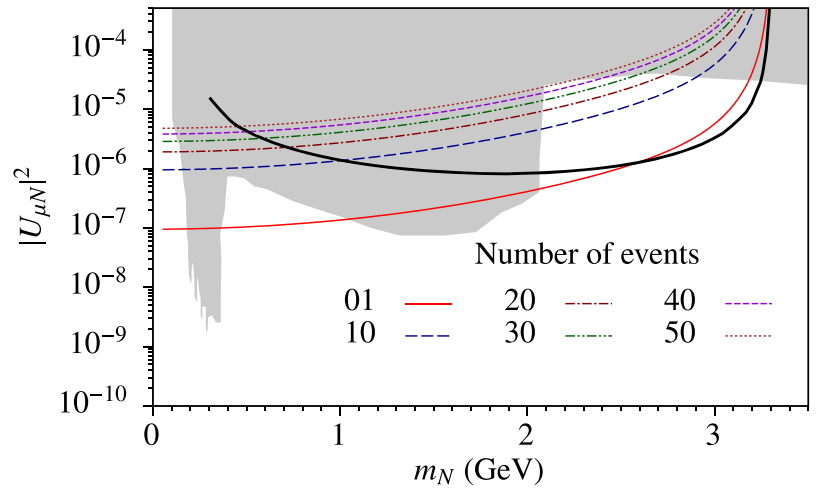

(a) From the decay $B \rightarrow D \mu N$.

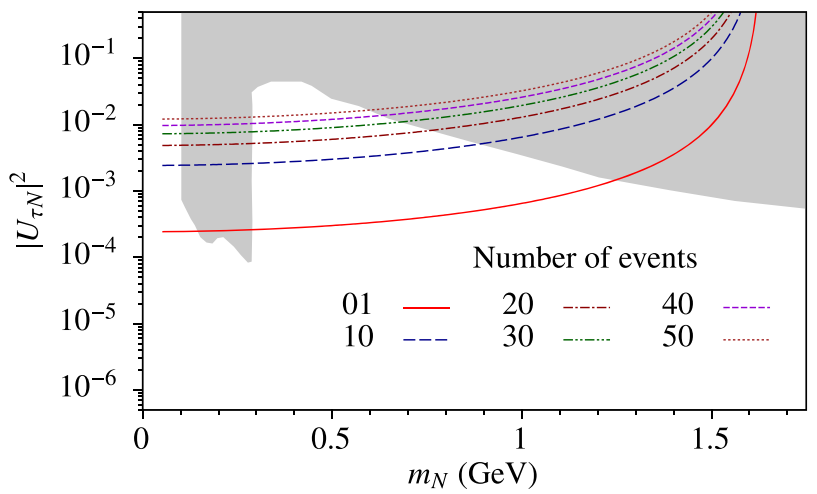

(b) From the decay $B \rightarrow D \tau N$.

Fig. 1 Values of $\left|U_{\mu N}\right|^{2}$ and $\left|U_{\tau N}\right|^{2}$ estimated from observed number of events (less than 50 events) of the decays $B \rightarrow D \mu N$ and $B \rightarrow$ $D \tau N$, respectively, using the projected number of $B$ decays at Belle II and assuming $0.1 \%$ chance of full reconstruction of $\tau$ from its decays. The $B \rightarrow D \ell N$ decays include both the charged and neutral modes. The thick solid line corresponds to the predicted 95\% C.L. upper-limit on $\left|U_{\mu N}\right|^{2}$ [36], for $4.8 \times 10^{12} B$ decay events at upgraded $\mathrm{LHCb}$ (with the decay $B \rightarrow D \mu \mu \pi$ )

where $\epsilon_{D}, \epsilon_{\ell}$ denote the efficiency to reconstruct the $D, \ell$ in the signal side. In our numerical study discussed below we have assumed $\epsilon_{D}=\epsilon_{\mu}=1$, but $\epsilon_{\tau}=0.001$ (the reason of which is given later).

For a numerical study we consider the decays $B \rightarrow D \mu N$ and $B \rightarrow D \tau N$ in context of Belle II experiment, which is poised to detect $10^{11} B$ decay events [108]. Out of these, about $0.61 \%$ of charged $B$ events and $0.34 \%$ of neutral $B$ events can be fully reconstructed from hadronic tagging [108], so that only about $4.8 \times 10^{8}$ events of $B$ decays get fully reconstructed. Considering only these $B$ decays, we are able to estimate the value of $\left|U_{\mu N}\right|^{2}$, as shown in Fig. 1a, from possible observation of 50 events or less for $B \rightarrow D \mu N$. It is easy to observe that in the mass range $\sim 2-3 \mathrm{GeV}$ our approach can provide stronger constraint, by about one order of magnitude, than the existing experimental upper-limit (exclusion region at $\sim 90 \%$ C.L. from various experiments is shown by the shaded region in gray). 
Figure 1a also shows that our constraint is comparable with the $95 \%$ C.L. upper-limit on $\left|U_{\mu N}\right|^{2}$, shown as a thick solid line, predicted in Ref. [36] based on $4.8 \times 10^{12} B$ decay events at upgraded $\mathrm{LHCb}$ (with the decay $B \rightarrow D \mu \mu \pi$ ). For $m_{N}<2 \mathrm{GeV}$ (important for light sterile neutrino searches) our constraint significantly surpasses the above-mentioned constraint predicted for $\mathrm{LHCb}$ upgrade. This is primarily due to the suppression factors affecting the observation of $B \rightarrow D \mu \mu \pi$ decays inside a finite-sized detector for smaller values of $m_{N}$ (see the next section and Figs. 4 and 6). It is worth mentioning that the method proposed in this paper cannot be applied to $\mathrm{LHCb}$, for it requires full reconstruction of the rest of the event so that missing energy-momentum can be used to extract the information about $N$.

Similarly, we can constrain $\left|U_{\tau N}\right|^{2}$ from number of observed $B \rightarrow D \tau N$ decays if the 4-momentum of the final $\tau$ could be measured accurately. In Fig. 1b, we show estimations of $\left|U_{\tau N}\right|^{2}$ as a function of $m_{N}$, for different values of observed $B \rightarrow D \tau N$ decay events. Here we have assumed that the 4-momenta of only $0.1 \%$ of all the $\tau$ decays could be precisely measured (e.g. more than 3-prong decays of $\tau$ [109]). It is clear from Fig. $1 \mathrm{~b}$ that our constraint on $\left|U_{\tau N}\right|^{2}$ in the mass range $[0.3,1] \mathrm{GeV}$ is more stringent than the existing studies, by an order of magnitude in some $m_{N}$ region. It should be noted that if we can further improve the $\tau$ reconstruction efficiency, our current result would further improve.

At this point, it is important to discuss how the signal events can be experimentally distinguished from possible SM background events. To illustrate our approach, we shall consider the signal processes $B \rightarrow D \mu N$ for $m_{N}=(1.0,2.0,3.0) \pm 0.1 \mathrm{GeV}$, the SM allowed process $B \rightarrow D \mu v_{\mu}$, and the SM background process $B \rightarrow$ $D \mu \nu_{\mu} \pi_{\text {soft }} / \gamma_{\text {soft }}$, where we have taken pions with energy $<0.2 \mathrm{GeV}$ and photons with energy $<0.1 \mathrm{GeV}$ in the rest frame of the $B$-meson as soft pions and soft photons respectively. If we consider the existing experimental limits on $\left|U_{\mu N}\right|^{2}$, then for the cases of $m_{N}=1.0,2.0,3.0 \mathrm{GeV}$ we expect to obtain about $1,1,6$ signal events, ${ }^{1}$ respectively, which are too few for any proper analysis. Besides, to keep our proposal for the new experimental search devoid of any prejudice and yet optimistic, let us fix the value of $\left|U_{\mu N}\right|^{2}$ at $10^{-5}$ for all values of $m_{N}$ under our consideration. This yields about 75, 25, 2 signal events for $m_{N}=1.0,2.0,3.0 \mathrm{GeV}$ cases, respectively. ${ }^{2}$ In Fig. 2 we compare the signal event distributions vs. the SM events and SM background events, with respect to the energy of muon $\left(E_{\mu}\right)$, the missing energy ( $E_{\text {miss }}$, with $E_{\text {miss }}=E_{N}$ for signal events), the missing mass ( $m_{\text {miss }}$, with $m_{\text {miss }}=m_{N}$ for signal events) and the invariant mass-square $s=\left(p_{B}-p_{D}\right)^{2}$. It is clear from Fig. 2 that

\footnotetext{
1 Those numbers of signal events can be easily seen from Fig. 1a.

2 See Fig. 2, and please note that we have considered for each mass $m_{N}$ the number of events $/ 0.1 \mathrm{GeV}$.
}

the missing mass distribution is the most useful one among all the observables, as the NP scenarios with $m_{N}>1 \mathrm{GeV}$ are easily discernible. Nevertheless, combinations of all the observables could be used to look for the sterile neutrino signature. Since the SM and SM background processes have much larger statistics and they are well understood both theoretically and experimentally, one could, in principle, implement multi-variate analysis or likelihood studies to figure out NP cases for $m_{N}<1 \mathrm{GeV}$.

We have demonstrated how the active-sterile mixing parameters can be probed without considering any sequential decay of sterile neutrino, provided the 4-momenta of all the other particles are well measured. However, as mentioned before and as is well known, the Dirac and Majorana nature of the sterile neutrino can be probed only when its sequential decay inside detector is considered.

\section{Probing the Dirac and Majorana nature of the neutrino $N$}

If the sterile neutrino $N$ decays inside a detector, we can probe lepton number violation in the entire process (which includes both the production of $N$ and its sequential decay) to ascertain its Majorana nature. As an example, let us consider the sequential decay, $B^{0} \rightarrow D^{-} \mu^{+} \mu^{\mp} \pi^{ \pm} \equiv$ $\left(B^{0} \rightarrow D^{-} \mu^{+} N\right) \otimes\left(N \rightarrow \mu^{\mp} \pi^{ \pm}\right)$. The meson-level Feynman diagrams for these decays are shown in Fig. 3. It is very clear that observation of the lepton number violating mode $B^{0} \rightarrow D^{-} \mu^{+} \mu^{+} \pi^{-}$would imply that the sterile neutrino has Majorana nature. While reconstructing the sterile neutrino from the final states $\mu^{\mp} \pi^{ \pm}$in the detector, we must also include an observable spatial separation between the point of production and the point of decay of the sterile neutrino. We can also consider the decay $N \rightarrow \tau^{\mp} \pi^{ \pm}$if allowed by kinematics. Similar analysis as above can be done for $D^{0} \rightarrow K^{-} \mu^{+} \mu^{\mp} \pi^{ \pm}$and related decays as well.

For a detector of finite size, say $L_{D}$, the observation of displaced vertex with decay length $L$ necessarily demands that $L<L_{D}$, and this depends on the lifetime and energy of the sterile neutrino.

The feasibility of studying the Dirac and Majorana signatures of the sterile neutrino $N$ (of mass $m_{N}$, energy $E_{N}$ and total decay rate $\Gamma_{N}$ ) via $B \rightarrow D \mu \mu \pi$ decays by using a detector of finite size $L_{D}$ depends on two important factors, (1) $P_{\text {decay }}(L)$, the probability of decay of $N$ within $L<L_{D}$, and (2) $P_{\text {flip }}$, the probability of helicity flip required for observation of the LNV decays which characterize the Majorana neutrino, and these are given by

$P_{\text {decay }}\left(m_{N}, E_{N}, L\right)=1-\exp \left(-L m_{N} \Gamma_{N} / \sqrt{E_{N}^{2}-m_{N}^{2}}\right)$, 


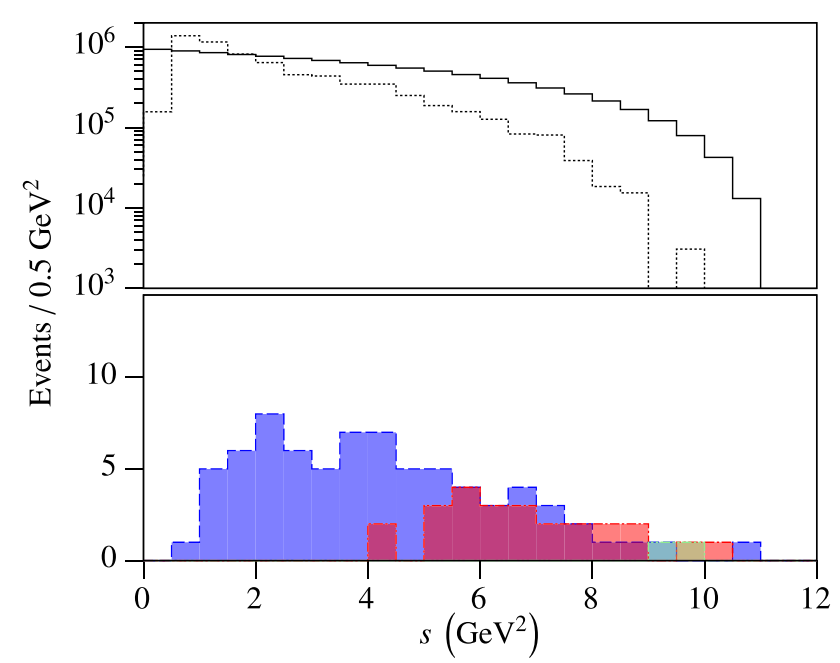

(a) Distribution of events with respect to $s$.

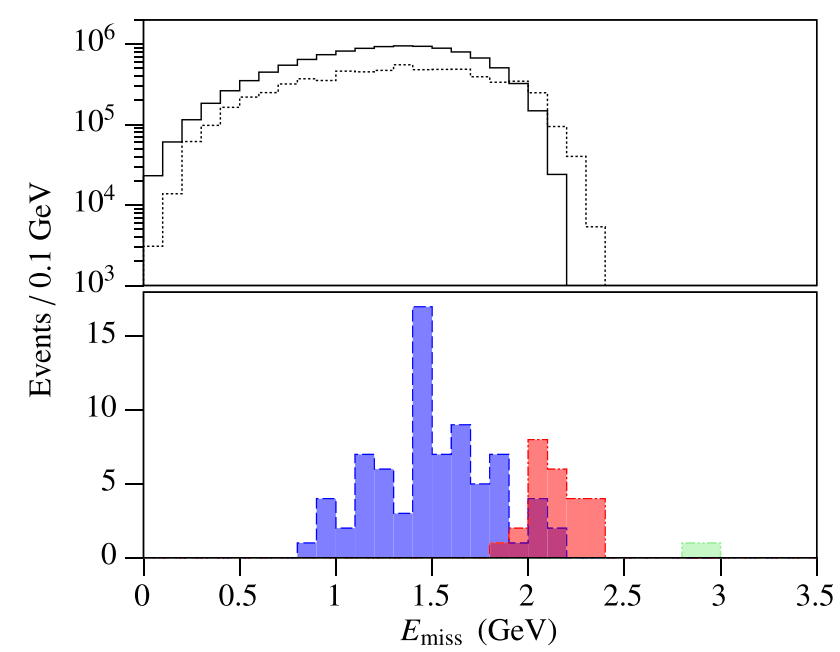

(c) Distribution of events with respect to $E_{\text {miss }}$.

Fig. 2 Distribution of events corresponding to SM process $B \rightarrow$ $D \mu v_{\mu}$, SM background process $B \rightarrow D \mu \nu_{\mu} \pi_{\text {soft }} / \gamma_{\text {soft }}$, and the new physics (NP) decays $B \rightarrow D \mu N$ for $m_{N}=(1.0,2.0,3.0) \pm 0.1 \mathrm{GeV}$, with respect to a few observables. Note that the number of events for

$$
P_{\text {flip }}\left(m_{N}, E_{N}\right)=m_{N}^{2} /\left(E_{N}+\sqrt{E_{N}^{2}-m_{N}^{2}}\right)^{2}
$$

We can quantify the feasibility of observing the full decay $B \rightarrow D \mu \mu \pi$ at Belle II, by the following distribution of events with respect to $E_{N}$,

$$
\begin{aligned}
\frac{d \mathcal{N}_{L N C}\left(m_{N}, E_{N}, L\right)}{d E_{N}}= & \frac{N_{B}}{\Gamma_{B}} \frac{d \Gamma(B \rightarrow D \mu N)}{d E_{N}} \times \operatorname{Br}(N \rightarrow \mu \pi) \\
& \times P_{\text {decay }}\left(m_{N}, E_{N}, L\right) \\
& \times \epsilon_{D} \times \epsilon_{\mu, 1} \times \epsilon_{\mu, 2} \times \epsilon_{\pi}, \\
\frac{d \mathcal{N}_{L N V}\left(m_{N}, E_{N}, L\right)}{d E_{N}}= & \frac{d \mathcal{N}_{L N C}\left(m_{N}, E_{N}, L\right)}{d E_{N}} \times P_{\text {flip }}\left(m_{N}, E_{N}\right),
\end{aligned}
$$

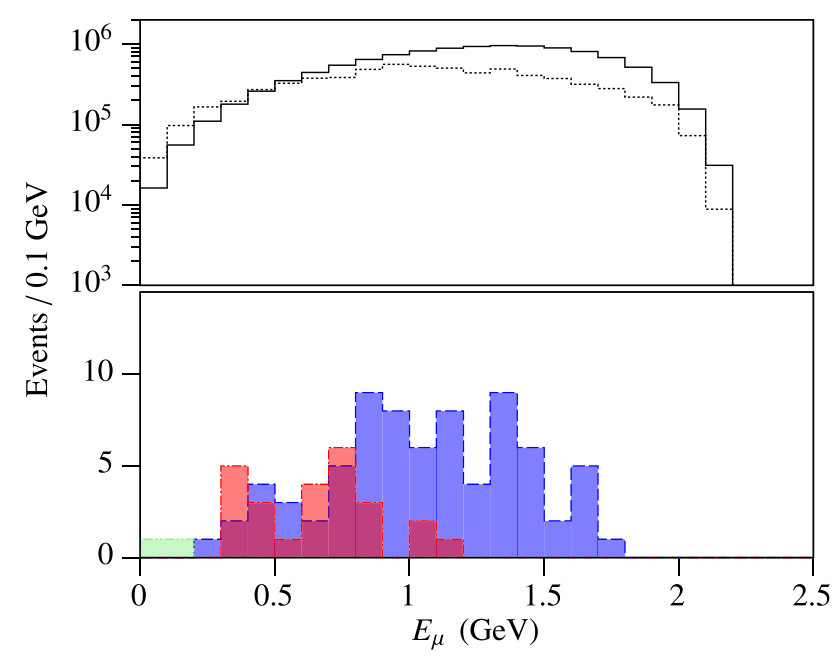

(b) Distribution of events with respect to $E_{\mu}$.

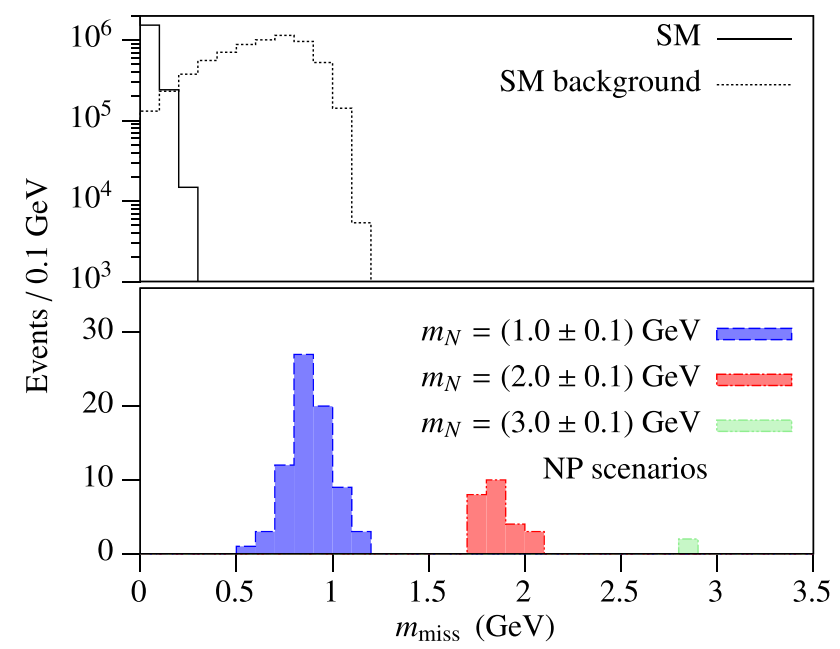

(d) Distribution of events with respect to $m_{\text {miss }}$.

SM and SM background processes are very large and, hence, those are shown with the vertical axis in log-scale, while the NP scenarios are shown in a linear scale

where $\mathcal{N}_{L N C}, \mathcal{N}_{L N V}$, respectively, denote the number of LNC and LNV events, $N_{B}$ is the total number of $B$ mesons produced/analyzed in the experiment, $\Gamma_{B}$ is the total decay rate of the $B$ meson, and $\epsilon_{D}, \epsilon_{\mu, 1}, \epsilon_{\mu, 2}, \epsilon_{\pi}$ are the various efficiency factors corresponding to the reconstruction of final $D$ meson, $\mu$ from the first vertex, $\mu$ from the second vertex and the $\pi$, respectively. For our numerical study we have assumed all these efficiency factors to be 1 . It should be noted that inclusion of $P_{\text {flip }}\left(m_{N}, E_{N}\right)$ is an effective way of introducing the helicity flip. The most accurate way is to consider the full decay $B \rightarrow D \mu \mu \pi$ along with the propagator for the intermediate $N$ and that would automatically lead to $m_{N}$ dependence (similar to the mass-dependence 


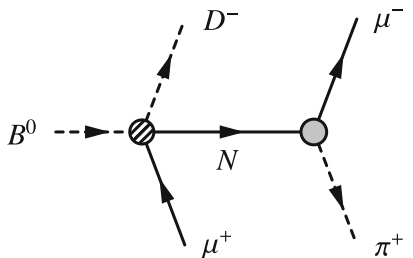

(a) Both Dirac and Majorana $N$

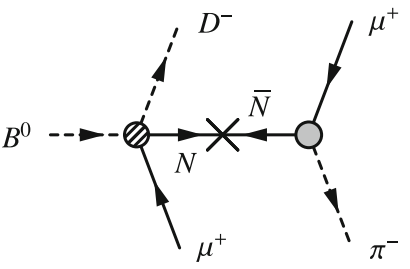

(b) Only Majorana $N$
Fig. 3 Meson-level Feynman diagrams contributing to the decays $B^{0} \rightarrow D^{-} \mu^{+} \mu^{\mp} \pi^{ \pm}$. The sterile neutrino is produced at the first vertex and decays at the second vertex, which is at an observable distance away from the first vertex. The circular blobs connote the contributions from the corresponding hadronic form factors and decay constants. The cross in the Majorana scenario denotes the helicity flip involved in the decay

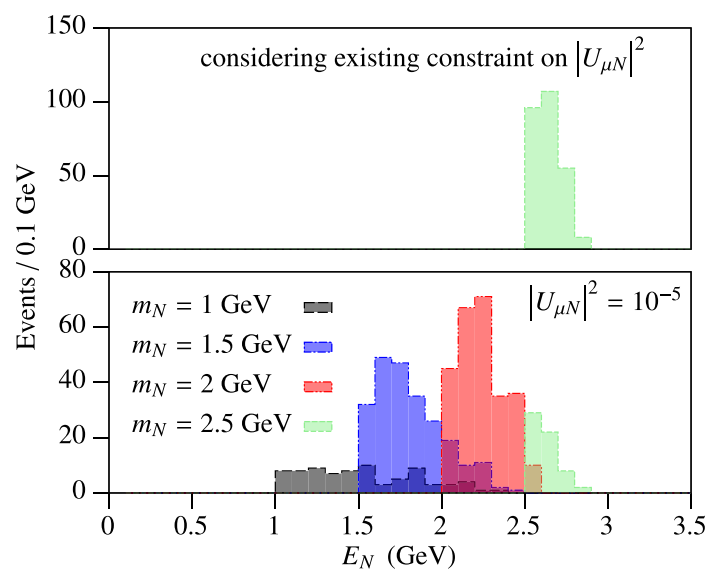

Fig. 4 Distribution of the number of events of the lepton number conserving $B \rightarrow D \mu^{ \pm} \mu^{\mp} \pi$ decays. Here we have considered the displaced vertices not larger than $1 \mathrm{~m}$ so that the events can be observed at Belle II

found in neutrinoless double-beta decay, a classic example of a LNV process). By including helicity-flip factor in $d \mathcal{N}_{L N V}\left(m_{N}, E_{N}, L\right) / d E_{N}$ it is clear that for $m_{N} \rightarrow 0$, the difference between Dirac and Majorana cases vanish as per the 'practical Dirac-Majorana confusion theorem' $[110,111]$.

In the numerical study shown in Fig. 4 we have studied the energy distribution of the $\mu \pi$ system originating from the decay of $N$. It is noticeable that for $m_{N} \leqslant 2 \mathrm{GeV}$ scenarios, no $B \rightarrow D \mu^{ \pm} \mu^{\mp} \pi$ decays with characteristic displaced vertices $\leqslant 1 \mathrm{~m}$ are expected to be observable at Belle II (considering the size of the central drift chamber [108]) if we consider the existing constraints on $\left|U_{\mu N}\right|^{2}$ (see Refs. [35,36] for details of existing constraints). However, if we consider $\left|U_{\mu N}\right|^{2}=10^{-5}$, the smaller mass scenarios are also feasible. It must be noted that the number of events shown here are larger than the ones shown in Fig. 2c simply because we have considered the full sample of $B$ mesons here whereas for Fig. 2c only the smaller number of fully reconstructed $B$ decays were considered. Figure 4 shows that as the mass of neutrino gets smaller the mean distance of displaced ver- tices gets bigger which would put most of the decays outside the Belle II detector, unless there is compensation by a substantial increase in the value of $\left|U_{\mu N}\right|^{2}$ which facilitates an appreciable number of events still happening inside the detector. This is also clearly discernible from Fig. 5 where we have varied the decay length $L$ within which the observed displaced vertices would lie. As per the existing constraint on $\left|U_{\mu N}\right|^{2}$ if we wish to observe $B \rightarrow D \mu^{\mp} \mu^{ \pm} \pi$ events in a detector for $m_{N} \leqslant 2 \mathrm{GeV}$, our existing detectors are clearly not suitable. Nevertheless, our approach elaborated in previous sections might come handy in the search for discovery of such sterile neutrino(s). It should be noted that the energy $E_{N}$ in both Figs. 4 and 5 are measured in the rest frame of the parent $B$ meson. Figure 5 also illustrates an interesting behavior that the probability of decaying within a smaller decay length is larger when the neutrino is less energetic or equivalently more non-relativistic. Moreover, as we go to higher masses, the neutrino decays faster with larger decay width and shorter life time.

Finally assuming that one can observe the full decays at Belle II, we can estimate the value of the active-sterile mixing parameter $\left|U_{\mu N}\right|^{2}$ as a function of observed number of events. In Fig. 6 we have analyzed how the number of events varies for different values of $m_{N}$ and $\left|U_{\mu N}\right|^{2}$. We have also considered the additional suppression from helicity flip factor $P_{\text {flip }}$ while considering the LNV mode. For the total decay rate of $N$ which enters $P_{\text {decay }}(L)$ we have used Eqs. (30-32) of Ref. [86] and it depends on $\left|U_{e N}\right|^{2},\left|U_{\mu N}\right|^{2}$ and $\left|U_{\tau N}\right|^{2}$. Since $\left|U_{e N}\right|^{2}$ is already constrained by $0 \nu \beta \beta$ experiments to be much smaller than $\left|U_{\mu N}\right|^{2}$ and $\left|U_{\tau N}\right|^{2}$ (see Ref. [35]), we can safely neglect its contribution. It can be inferred from Fig. 6 that for $\left|U_{\mu N}\right|^{2}$ smaller than the existing experimental upper limit, one can still aspire to observe more than a handful of $B \rightarrow D \mu \mu \pi$ decays at Belle II with displaced vertex signatures.

\section{Conclusion}

In this paper we offer a new strategy that could lead to the discovery of a heavy sterile neutrino $N$ of mass $m_{N} \leqslant 3.3 \mathrm{GeV}$ and having appreciable mixing with active neutrinos, at the Belle II experiment. Consideration of the defining properties of a sterile neutrino such as it being an electrically neutral, spin- $1 / 2$ fermion with non-zero mass and possibly long lifetime, irrespective of its Dirac or Majorana nature, points out that decays such as $B \rightarrow D \ell N$, with $\ell=\mu, \tau$ and without considering the sequential decay of $N$, could play a crucial role in the discovery of $N$. Once $N$ is discovered, it is possible to probe the Majorana nature via searching for the lepton number violating processes.

We have shown that at Belle II, with only about $4.8 \times 10^{8}$ events of fully reconstructed $B \rightarrow D \mu N$ decays, one can 

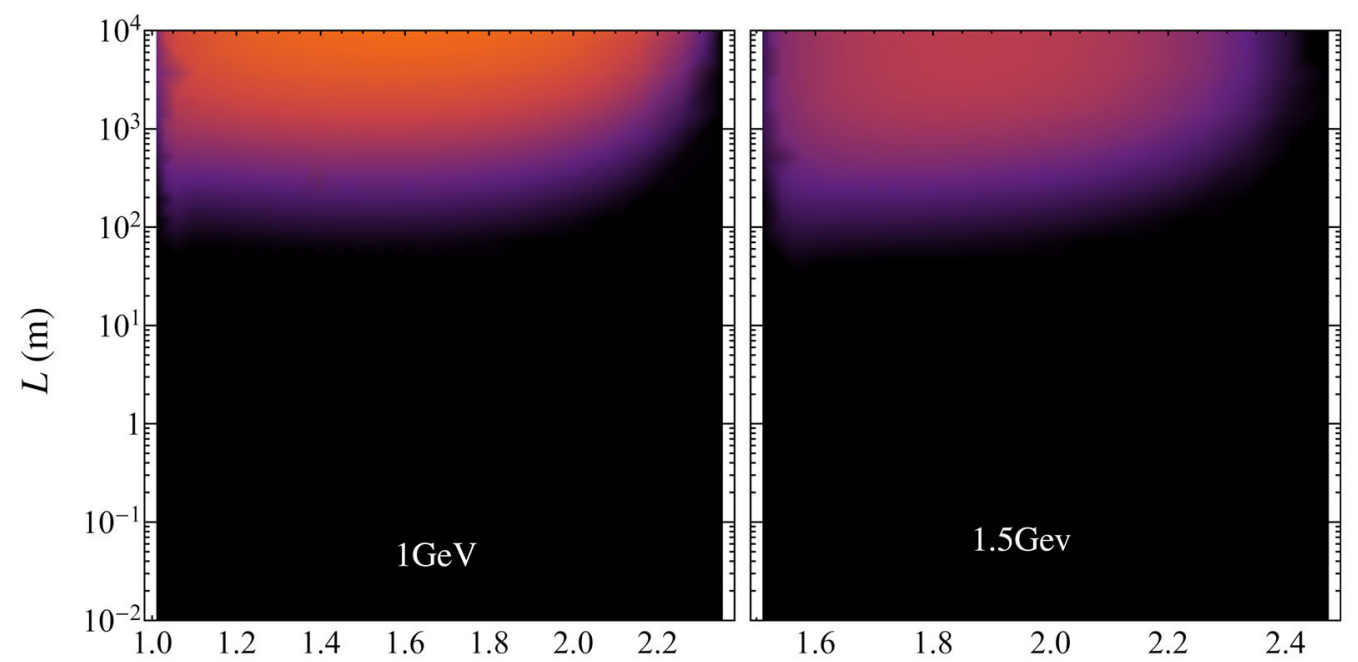

$10^{4}$
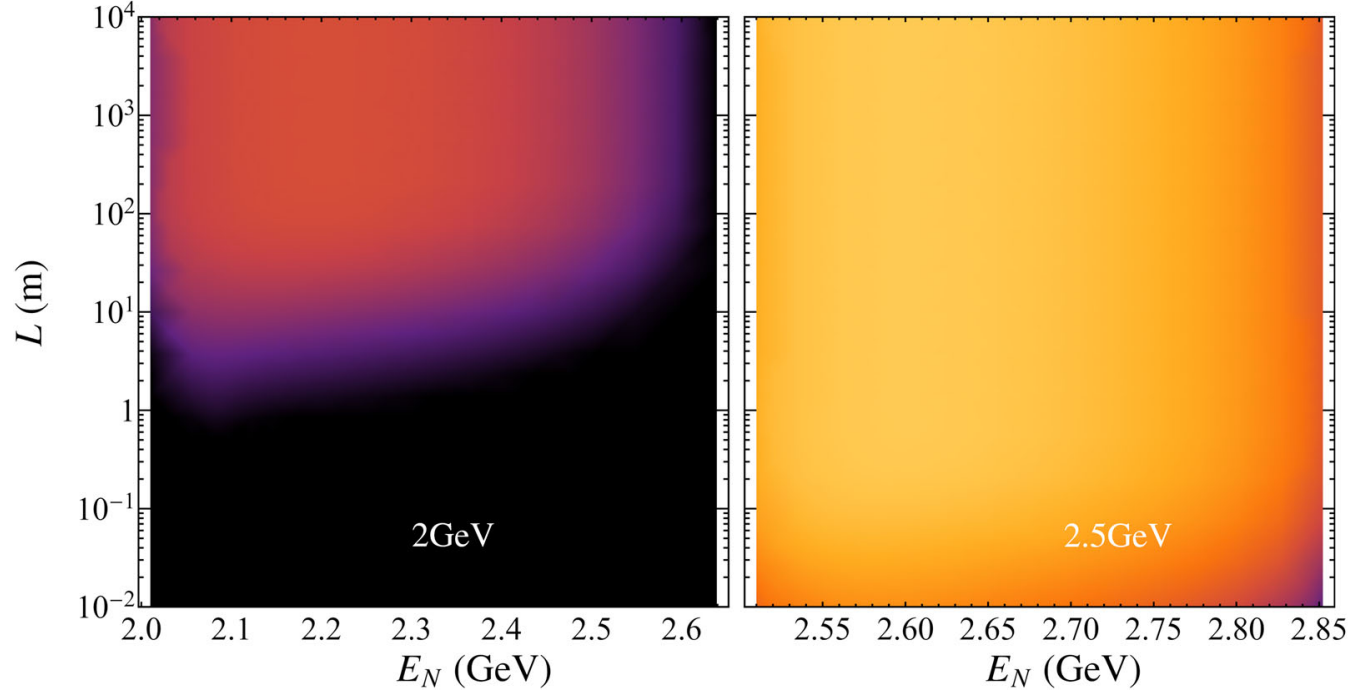

for observation at Belle II. We have considered four benchmark cases corresponding to $m_{N}=1,1.5,2,2.5 \mathrm{GeV}$. The neutrino $N$ is assumed to have Dirac nature and the existing constraint on $\left|U_{\mu N}\right|^{2}$ is considered here. The black colored region corresponds to number of events $\leqslant 1$ displaced vertices of lengths. Here the decays happen within the distance $L$ and only those decays with $L \leqslant 1 \mathrm{~m}$ are currently feasible

probe the mixing parameter $\left|U_{\mu N}\right|^{2}$ to a precision which is comparable with what $\mathrm{LHCb}$ can probe with about $4.8 \times 10^{12}$ events of $B \rightarrow D^{(*)} \mu \mu \pi$ decays. Our approach exploits the fact that at Belle II the 4-momentum of the parent $B$ meson decaying to $D \mu N$ can be inferred from the 4-momentum of the accompanying $\bar{B}$ meson, a feat not feasible at LHCb. It is noteworthy that for $m_{N}>1.0 \mathrm{GeV}$, it is easy to remove possible SM background contamination. For $m_{N}<1.0 \mathrm{GeV}$, a more refined analysis of SM background would be helpful in extracting the signal events.

It is certainly alluring to consider the full decay final states of $B \rightarrow D \mu \mu \pi$ as they can be used to probe Dirac or Majorana nature of $N$. However, these decays can have other new physics contributions in addition to contribution from $N$. Considering the contribution from $N$ as the major one, such decays still suffer suppression from displaced vertices that must lie within the finite sized detectors, as well as from branching ratio of the sequential $N$ decay and possible spinflip associated with the Majorana signature. Thus, despite their undisputed strength, these suppressed decays would be difficult to observe and discovery of $N$ might be missed if such decays are considered. For some masses, especially the heavier mass of $N$, however, we can still expect to observe a few such events even at Belle II, albeit the lower number of $B$ mesons produced as compared to $\mathrm{LHCb}$.

In summary, in this paper we propose the most systematic approach to probe a sterile neutrino $N$ of mass $m_{N} \leqslant 3.3$ $\mathrm{GeV}$, by using the decays $B \rightarrow D \ell N$ (with $\ell=\mu, \tau$ ) rather than the suppressed $B \rightarrow \ell_{1} \ell_{2} \pi, X \ell_{1} \ell_{2} \pi$ decays (with $\ell_{1,2}=e, \mu$ and $X=\pi, D$ ) previously used at Belle and 
Fig. 6 Numerical study of feasibility of observing Dirac (LNC) signal $B \rightarrow D \mu^{ \pm} \mu^{\mp} \pi$ and Majorana (LNV) signal $B \rightarrow D \mu^{ \pm} \mu^{ \pm} \pi$, inside Belle II detector with decay lengths less than $1 \mathrm{~m}$. Here we have neglected the contributions from both $\left|U_{e N}\right|^{2}$ and $\left|U_{\tau N}\right|^{2}$ when compared with $\left|U_{\mu N}\right|^{2}$
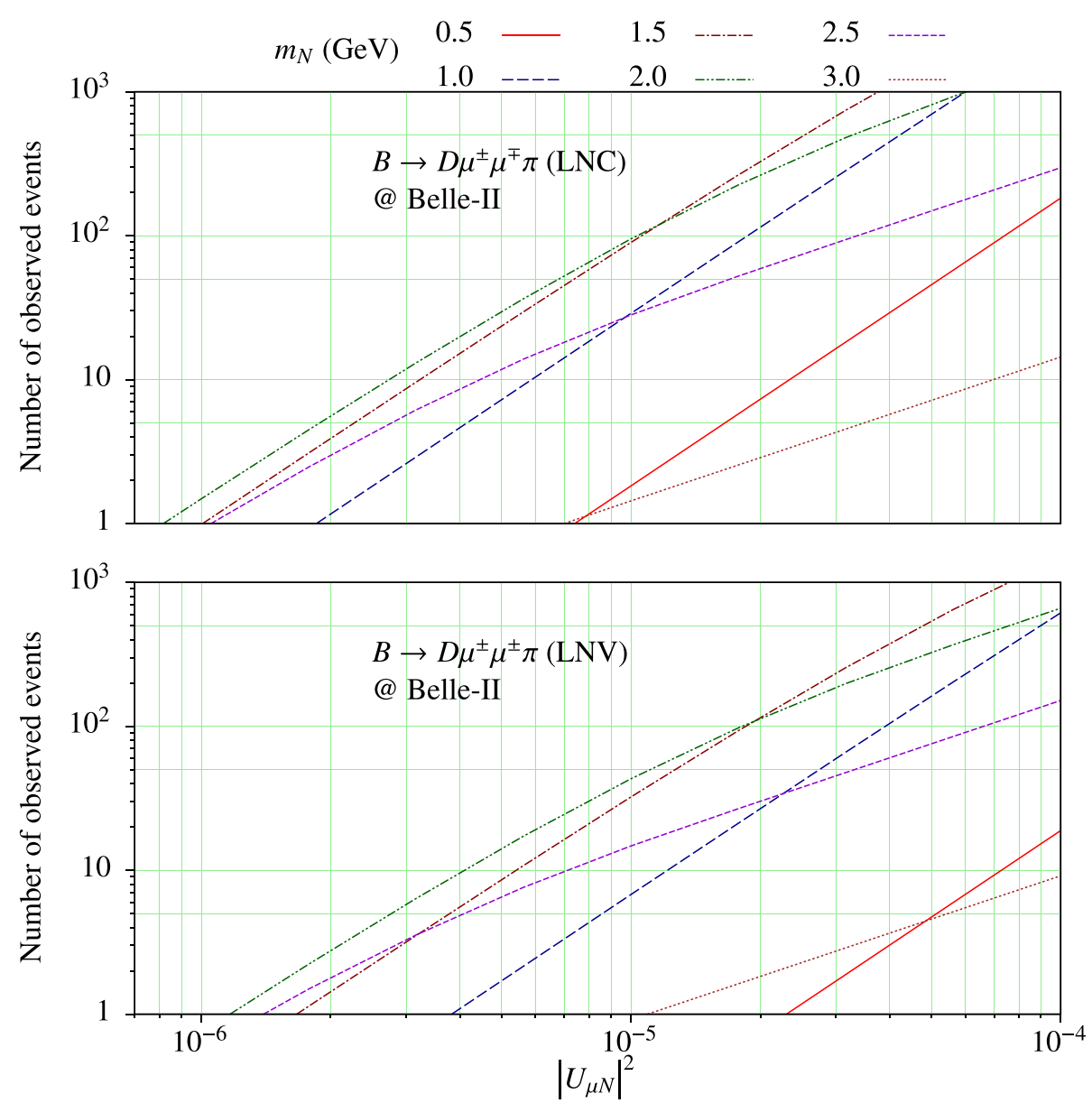

LHCb $[112,113]$. Our proposal does not require the sequential decay of $N$. The constraint on $\left|U_{\mu N}\right|^{2}$ thus achievable from Belle II is not only better than the existing experimental constraints in certain mass range of $m_{N}$ (viz. $0.4-1 \mathrm{GeV}$ and $2-3 \mathrm{GeV}$ ), but also better than the constraint that is achievable at upgraded LHCb for $m_{N}<2 \mathrm{GeV}$. The minimum value of $m_{N}$ that can be probed is constrained only by experimental accuracy of measurement of 4-momenta of $B, D$ and $\mu$. The sequential decay of $N$, useful to distinguish its Dirac or Majorana nature by observing the LNC or LNV modes respectively, is suppressed from observation of displaced vertices (for both LNC and LNV cases) and helicity flip (for LNV case only). Our numerical study shows if no decays of $N$ get observed within decay lengths $\leqslant 1 \mathrm{~m}$, the existing experimental upper limit on $\left|U_{\mu N}\right|^{2}$ can be improved.

Acknowledgements The work of C.S.K. was supported in part by the National Research Foundation of Korea (NRF) grant funded by the Korean government (MSIP) (NRF2018R1A4A1025334). This work of D.S. was supported (in part) by the Yonsei University Research Fund (Post Doc. Researcher Supporting Program) of 2018 (project no.: 201812-0145).

Data Availability Statement This manuscript has no associated data or the data will not be deposited. [Authors' comment: This is a theo- retical study and sources of all experimental data used are cited at the appropriate places in the article.]

Open Access This article is licensed under a Creative Commons Attribution 4.0 International License, which permits use, sharing, adaptation, distribution and reproduction in any medium or format, as long as you give appropriate credit to the original author(s) and the source, provide a link to the Creative Commons licence, and indicate if changes were made. The images or other third party material in this article are included in the article's Creative Commons licence, unless indicated otherwise in a credit line to the material. If material is not included in the article's Creative Commons licence and your intended use is not permitted by statutory regulation or exceeds the permitted use, you will need to obtain permission directly from the copyright holder. To view a copy of this licence, visit http://creativecomm ons.org/licenses/by/4.0/.

Funded by $\mathrm{SCOAP}^{3}$.

\section{References}

1. P. Minkowski, Phys. Lett. B 67, 421 (1977). https://doi.org/10. 1016/0370-2693(77)90435-X

2. M. Gell-Mann, P. Ramond, R. Slansky, in Sanibel Conference, "The Family Group in Grand Unified Theories", Feb. 1979, CALT-68-709 (1979), reprinted in arXiv:hep-ph/9809459

3. M. Gell-Mann, P. Ramond, R. Slansky, "Complex Spinors and Unified Theories", Print 80-0576, published, in Supergravity, ed. 
by D. Freedman, et al. (North-Holland, Amsterdam, 1979). KEK79-18-95

4. T. Yanagida, Conf. Proc. C 7902131, 95 (1979)

5. Glashow S.L., The Future of Elementary Particle Physics. in Quarks and Leptons. NATO Advanced Study Institutes Series (Series B. Physics), ed. by M. Lévy, J.L. Basdevant, D. Speiser, J. Weyers, R. Gastmans, M. Jacob. M. vol. 61 (Springer, Boston, 1980). https://doi.org/10.1007/978-1-4684-7197-7_15

6. R.N. Mohapatra, G. Senjanović, Phys. Rev. Lett. 44, 912 (1980). https://doi.org/10.1103/PhysRevLett.44.912

7. D. Wyler, L. Wolfenstein, Nucl. Phys. B 218, 205 (1983). https:// doi.org/10.1016/0550-3213(83)90482-0

8. E. Witten, Nucl. Phys. B 258, 75 (1985). https://doi.org/10.1016/ 0550-3213(85)90603-0

9. R.N. Mohapatra, J.W.F. Valle, Phys. Rev. D 34, 1642 (1986). https://doi.org/10.1103/PhysRevD.34.1642

10. A. Pilaftsis, T.E.J. Underwood, Phys. Rev. D 72, 113001 (2005). https://doi.org/10.1103/PhysRevD.72.113001

11. M. Malinsky, J.C. Romao, J.W.F. Valle, Phys. Rev. Lett. 95, 161801 (2005). https://doi.org/10.1103/PhysRevLett.95.161801

12. P.S.B. Dev, R.N. Mohapatra, Phys. Rev. D 81, 013001 (2010). https://doi.org/10.1103/PhysRevD.81.013001

13. P.S.B. Dev, A. Pilaftsis, Phys. Rev. D 86, 113001 (2012). https:// doi.org/10.1103/PhysRevD.86.113001

14. C.H. Lee, P.S. Bhupal Dev, R.N. Mohapatra, Phys. Rev. D 88(9), 093010 (2013). https://doi.org/10.1103/PhysRevD.88.093010

15. A. Das, N. Okada, Phys. Rev. D 88, 113001 (2013). https://doi. org/10.1103/PhysRevD.88.113001

16. A. Das, P.S. Bhupal Dev, N. Okada, Phys. Lett. B 735, 364 (2014). https://doi.org/10.1016/j.physletb.2014.06.058

17. W. Buchmüller, C. Greub, Nucl. Phys. B 363, 345 (1991). https:// doi.org/10.1016/0550-3213(91)80024-G

18. T. Appelquist, R. Shrock, Phys. Lett. B 548, 204 (2002). https:// doi.org/10.1016/S0370-2693(02)02854-X

19. T. Appelquist, R. Shrock, Phys. Rev. Lett. 90, 201801 (2003). https://doi.org/10.1103/PhysRevLett.90.201801

20. T. Appelquist, M. Piai, R. Shrock, Phys. Rev. D 69, 015002 (2004). https://doi.org/10.1103/PhysRevD.69.015002

21. J. Kersten, A.Y. Smirnov, Phys. Rev. D 76, 073005 (2007). https:// doi.org/10.1103/PhysRevD.76.073005

22. F. del Aguila, J.A. Aguilar-Saavedra, J. de Blas, M. Zralek, Acta Phys. Pol. B 38, 3339 (2007)

23. X.G. He, S. Oh, J. Tandean, C.C. Wen, Phys. Rev. D 80, 073012 (2009). https://doi.org/10.1103/PhysRevD.80.073012

24. A. Ibarra, E. Molinaro, S.T. Petcov, JHEP 1009, 108 (2010). https://doi.org/10.1007/JHEP09(2010)108

25. T. Asaka, S. Blanchet, M. Shaposhnikov, Phys. Lett. B 631, 151 (2005). https://doi.org/10.1016/j.physletb.2005.09.070

26. T. Asaka, M. Shaposhnikov, Phys. Lett. B 620, 17 (2005). https:// doi.org/10.1016/j.physletb.2005.06.020

27. D. Gorbunov, M. Shaposhnikov, JHEP 0710, 015 (2007). https:// doi.org/10.1088/1126-6708/2007/10/015 Erratum: [JHEP 1311, 101 (2013)]. https://doi.org/10.1007/JHEP11(2013)101

28. A. Boyarsky, O. Ruchayskiy, M. Shaposhnikov, Ann. Rev. Nucl. Part. Sci. 59, 191 (2009). https://doi.org/10.1146/annurev.nucl. 010909.083654

29. L. Canetti, M. Drewes, M. Shaposhnikov, Phys. Rev. Lett. 110(6), 061801 (2013). https://doi.org/10.1103/PhysRevLett. 110.061801

30. L. Canetti, M. Drewes, T. Frossard, M. Shaposhnikov, Phys. Rev. D 87(9), 093006 (2013). https://doi.org/10.1103/PhysRevD.87. 093006

31. L. Canetti, M. Drewes, B. Garbrecht, Phys. Rev. D 90(12), 125005 (2014). https://doi.org/10.1103/PhysRevD.90.125005

32. M. Drewes, B. Garbrecht, Nucl. Phys. B 921, 250 (2017). https:// doi.org/10.1016/j.nuclphysb.2017.05.001
33. S. Dodelson, L.M. Widrow, Phys. Rev. Lett. 72, 17 (1994). https:// doi.org/10.1103/PhysRevLett.72.17

34. A. Palazzo, Mod. Phys. Lett. A 28, 1330004 (2013). https://doi. org/10.1142/S0217732313300048

35. F.F. Deppisch, P.S. Bhupal Dev, A. Pilaftsis, New J. Phys. 17(7), 075019 (2015). https://doi.org/10.1088/1367-2630/17/7/075019

36. G. Cvetič, C.S. Kim, Phys. Rev. D 100(1), 015014 (2019). https:// doi.org/10.1103/PhysRevD.100.015014

37. W.H. Furry, Phys. Rev. 56, 1184 (1939). https://doi.org/10.1103/ PhysRev.56.1184

38. H. Primakoff, S.P. Rosen, Rep. Prog. Phys. 22, 121 (1959). https:// doi.org/10.1088/0034-4885/22/1/305

39. H. Primakoff, S.P. Rosen, Annu. Rev. Nucl. Part. Sci. 31, 145 (1981). https://doi.org/10.1146/annurev.ns.31.120181.001045

40. M. Doi, T. Kotani, E. Takasugi, Prog. Theor. Phys. Suppl. 83, 1 (1985). https://doi.org/10.1143/PTPS.83.1

41. S.R. Elliott, J. Engel, J. Phys. G 30, R183 (2004). https://doi.org/ 10.1088/0954-3899/30/9/R01

42. W. Rodejohann, Int. J. Mod. Phys. E 20, 1833 (2011). https://doi. org/10.1142/S0218301311020186

43. W.-Y. Keung, G. Senjanović, Phys. Rev. Lett. 50, 1427 (1983). https://doi.org/10.1103/PhysRevLett.50.1427

44. V. Tello, M. Nemevšek, F. Nesti, G. Senjanović, F. Vissani, Phys. Rev. Lett. 106, 151801 (2011). https://doi.org/10.1103/ PhysRevLett.106.151801

45. M. Nemevšek, F. Nesti, G. Senjanović, V. Tello, arXiv:1112.3061 [hep-ph]

46. S. Kovalenko, Z. Lu, I. Schmidt, Phys. Rev. D 80, 073014 (2009). https://doi.org/10.1103/PhysRevD.80.073014

47. G. Senjanović, Riv. Nuovo Cim. 34, 1 (2011). https://doi.org/10. 1393/ncr/i2011-10061-8

48. C.Y. Chen, P.S. Bhupal Dev, Phys. Rev. D 85, 093018 (2012). https://doi.org/10.1103/PhysRevD.85.093018

49. C.Y. Chen, P.S. Bhupal Dev, R.N. Mohapatra, Phys. Rev. D 88 033014 (2013). https://doi.org/10.1103/PhysRevD.88.033014

50. P.S. Bhupal Dev, A. Pilaftsis, U.k. Yang, Phys. Rev. Lett. 112, 081801 (2014). https://doi.org/10.1103/PhysRevLett.112. 081801

51. D. Alva, T. Han, R. Ruiz, JHEP 1502, 072 (2015). https://doi.org/ 10.1007/JHEP02(2015)072

52. A. Das, N. Okada, Phys. Rev. D 93(3), 033003 (2016). https:// doi.org/10.1103/PhysRevD.93.033003

53. C. Degrande, O. Mattelaer, R. Ruiz, J. Turner, Phys. Rev. D 94(5), 053002 (2016). https://doi.org/10.1103/PhysRevD.94.053002

54. A. Das, P. Konar, S. Majhi, JHEP 1606, 019 (2016). https://doi. org/10.1007/JHEP06(2016)019

55. A. Das, arXiv:1701.04946 [hep-ph]

56. M. Kohda, H. Sugiyama, K. Tsumura, Phys. Lett. B 718, 1436 (2013). https://doi.org/10.1016/j.physletb.2012.12.048

57. J.C. Helo, M. Hirsch, S. Kovalenko, Phys. Rev. D 89, 073005 (2014). https://doi.org/10.1103/PhysRevD.89.073005 Erratum: [Phys. Rev. D 93, no. 9, 099902 (2016)]. https://doi.org/10.1103/ PhysRevD.93.099902

58. C.O. Dib, C.S. Kim, Phys. Rev. D 92(9), 093009 (2015). https:// doi.org/10.1103/PhysRevD.92.093009

59. C.O. Dib, C.S. Kim, K. Wang, J. Zhang, Phys. Rev. D 94(1), 013005 (2016). https://doi.org/10.1103/PhysRevD.94.013005

60. C.O. Dib, C.S. Kim, K. Wang, Phys. Rev. D 95(11), 115020 (2017). https://doi.org/10.1103/PhysRevD.95.115020

61. C.O. Dib, C.S. Kim, K. Wang, Chin. Phys. C 41(10), 103103 (2017). https://doi.org/10.1088/1674-1137/41/10/103103

62. A. Das, P.S.B. Dev, C.S. Kim, Phys. Rev. D 95(11), 115013 (2017). https://doi.org/10.1103/PhysRevD.95.115013

63. A. Das, Y. Gao, T. Kamon, Eur. Phys. J. C 79(5), 424 (2019). https://doi.org/10.1140/epjc/s10052-019-6937-7 
64. V. Gribanov, S. Kovalenko, I. Schmidt, Nucl. Phys. B 607, 355 (2001). https://doi.org/10.1016/S0550-3213(01)00169-9

65. G. Cvetič, C. Dib, C.S. Kim, J.D. Kim, Phys. Rev. D 66, 034008 (2002). https://doi.org/10.1103/PhysRevD.66.034008 Erratum: [Phys. Rev. D 68, 059901 (2003)]. https://doi.org/10.1103/ PhysRevD.68.059901

66. J.C. Helo, S. Kovalenko, I. Schmidt, Phys. Rev. D 84, 053008 (2011). https://doi.org/10.1103/PhysRevD.84.053008

67. J. Zamora-Saá, JHEP 1705, 110 (2017). https://doi.org/10.1007/ JHEP05(2017) 110

68. N. Shimizu [Belle Collaboration], PoS FPCP 2016, 022 (2017). https://doi.org/10.22323/1.280.0022

69. C.S. Kim, G. López Castro, D. Sahoo, Phys. Rev. D 96(7), 075016 (2017). https://doi.org/10.1103/PhysRevD.96.075016

70. L.S. Littenberg, R.E. Shrock, Phys. Rev. Lett. 68, 443 (1992). https://doi.org/10.1103/PhysRevLett.68.443

71. L.S. Littenberg, R.E. Shrock, Phys. Lett. B 491, 285 (2000). https://doi.org/10.1016/S0370-2693(00)01041-8

72. C. Dib, V. Gribanov, S. Kovalenko, I. Schmidt, Phys. Lett. B 493 , 82 (2000). https://doi.org/10.1016/S0370-2693(00)01134-5

73. A. Ali, A.V. Borisov, N.B. Zamorin, Eur. Phys. J. C 21, 123 (2001). https://doi.org/10.1007/s100520100702

74. M.A. Ivanov, S.G. Kovalenko, Phys. Rev. D 71, 053004 (2005). https://doi.org/10.1103/PhysRevD.71.053004

75. A. de Gouvea, J. Jenkins, Phys. Rev. D 77, 013008 (2008). https:// doi.org/10.1103/PhysRevD.77.013008

76. N. Quintero, G. López Castro, D. Delepine, Phys. Rev. D 84, 096011 (2011). https://doi.org/10.1103/PhysRevD.84. 096011 Erratum: [Phys. Rev. D 86, 079905 (2012)]. https://doi. org/10.1103/PhysRevD.86.079905

77. A. Abada, A.M. Teixeira, A. Vicente, C. Weiland, JHEP 1402, 091 (2014). https://doi.org/10.1007/JHEP02(2014)091

78. Y. Wang, S.S. Bao, Z.H. Li, N. Zhu, Z.G. Si, Phys. Lett. B 736, 428 (2014). https://doi.org/10.1016/j.physletb.2014.08.006

79. J.C. Helo, S. Kovalenko, I. Schmidt, Nucl. Phys. B 853, 80 (2011). https://doi.org/10.1016/j.nuclphysb.2011.07.020

80. A. Atre, T. Han, S. Pascoli, B. Zhang, JHEP 0905, 030 (2009). https://doi.org/10.1088/1126-6708/2009/05/030

81. G. Cvetič, C. Dib, S.K. Kang, C.S. Kim, Phys. Rev. D 82, 053010 (2010). https://doi.org/10.1103/PhysRevD.82.053010

82. G. Cvetič, C. Dib, C.S. Kim, JHEP 1206, 149 (2012). https://doi. org/10.1007/JHEP06(2012)149. [arXiv:1203.0573 [hep-ph]]

83. G. Cvetič, C.S. Kim, J. Zamora-Saá, J. Phys. G 41, 075004 (2014). https://doi.org/10.1088/0954-3899/41/7/075004

84. G. Cvetič, C. Dib, C.S. Kim, J. Zamora-Saá, Symmetry 7, 726 (2015). https://doi.org/10.3390/sym7020726

85. S. Mandal, N. Sinha, Phys. Rev. D 94(3), 033001 (2016). https:// doi.org/10.1103/PhysRevD.94.033001

86. G. Cvetič, C.S. Kim, Phys. Rev. D 94(5), 053001 (2016). https:// doi.org/10.1103/PhysRevD.94.053001 Erratum: [Phys. Rev. D 95, no. 3, 039901 (2017)]. https://doi.org/10.1103/PhysRevD.95. 039901

87. G. Cvetič, F. Halzen, C.S. Kim, S. Oh, Chin. Phys. C 41(11), 113102 (2017). https://doi.org/10.1088/1674-1137/41/ $11 / 113102$

88. R.E. Shrock, Phys. Lett. 96B, 159 (1980). https://doi.org/10.1016/ 0370-2693(80)90235-X

89. R.E. Shrock, Phys. Rev. D 24, 1232 (1981). https://doi.org/10. 1103/PhysRevD.24.1232
90. L. Lello, D. Boyanovsky, Phys. Rev. D 87, 073017 (2013). https:// doi.org/10.1103/PhysRevD.87.073017

91. G. Azuelos et al., Phys. Rev. Lett. 56, 2241 (1986). https://doi. org/10.1103/PhysRevLett.56.2241

92. N. De Leener-Rosier et al., Phys. Rev. D 43, 3611 (1991). https:// doi.org/10.1103/PhysRevD.43.3611

93. D.I. Britton et al., Phys. Rev. Lett. 68, 3000 (1992). https://doi. org/10.1103/PhysRevLett.68.3000

94. D.I. Britton et al., Phys. Rev. D 46, R885 (1992). https://doi.org/ 10.1103/PhysRevD.46.R885

95. M. Aoki et al. [PIENU Collaboration]. Phys. Rev. D 84, 052002 (2011). https://doi.org/10.1103/PhysRevD.84.052002

96. R. Abela, M. Daum, G.H. Eaton, R. Frosch, B. Jost, P.R. Kettle, E. Steiner, Phys. Lett. 105B, 263 (1981). https://doi.org/10.1016/ 0370-2693(81)90884-4 Erratum: [Phys. Lett. 106B, 513 (1981)]. https://doi.org/10.1016/0370-2693(81)90269-0

97. R.C. Minehart, K.O.H. Ziock, R. Marshall, W.A. Stephens, M. Daum, B. Jost, P.R. Kettle, Phys. Rev. Lett. 52, 804 (1984). https:// doi.org/10.1103/PhysRevLett.52.804

98. M. Daum, B. Jost, R.M. Marshall, R.C. Minehart, W.A. Stephens, K.O.H. Ziock, Phys. Rev. D 36, 2624 (1987). https://doi.org/10. 1103/PhysRevD.36.2624

99. D.A. Bryman, T. Numao, Phys. Rev. D 53, 558 (1996). https:// doi.org/10.1103/PhysRevD.53.558

100. K. Assamagan, C. Bronnimann, M. Daum, R. Frosch, P.R. Kettle, C. Wigger, Phys. Lett. B 434, 158 (1998). https://doi.org/10.1016/ S0370-2693(98)00727-8

101. T. Yamazaki et al., Conf. Proc. C 840719, 262 (1984). https:// inspirehep.net/files/559110f86c0e699c33e2c7911cd1c1fb

102. Y. Asano et al., Phys. Lett. 104B, 84 (1981). https://doi.org/10. 1016/0370-2693(81)90860-1

103. R.S. Hayano et al., Phys. Rev. Lett. 49, 1305 (1982). https://doi. org/10.1103/PhysRevLett.49.1305

104. A. Kusenko, S. Pascoli, D. Semikoz, JHEP 0511, 028 (2005). https://doi.org/10.1088/1126-6708/2005/11/028

105. A.V. Artamonov et al. [E949 Collaboration], Phys. Rev. D 91(5), 052001 (2015). https://doi.org/10.1103/PhysRevD.91. 052001 Erratum: [Phys. Rev. D 91, no. 5, 059903 (2015). https:// doi.org/10.1103/PhysRevD.91.059903]

106. C.S. Kim, G. López Castro, D. Sahoo, Phys. Rev. D 98(11), 115021 (2018). https://doi.org/10.1103/PhysRevD.98.115021

107. D.A. Bryman, R. Shrock, Phys. Rev. D 100(5), 053006 (2019). https://doi.org/10.1103/PhysRevD.100.053006

108. E. Kou et al. [Belle-II Collaboration], PTEP 2019(12), 123C01 (2019). https://doi.org/10.1093/ptep/ptz106

109. M. Tanabashi et al. [Particle Data Group], Phys. Rev. D 98(3), 030001 (2018). https://doi.org/10.1103/PhysRevD.98.030001

110. B. Kayser, R.E. Shrock, Phys. Lett. B 112, 137-142 (1982). https://doi.org/10.1016/0370-2693(82)90314-8

111. B. Kayser, Phys. Rev. D 26, 1662 (1982). https://doi.org/10.1103/ PhysRevD.26.1662

112. D. Liventsev et al. [Belle], Phys. Rev. D 87(7), 071102 (2013). https://doi.org/10.1103/PhysRevD.87.071102

113. R. Aaij et al. [LHCb], Phys. Rev. Lett. 112(13), 131802 (2014). https://doi.org/10.1103/PhysRevLett.112.131802 